\title{
The Influence of Centrifugal Force on the Interference Fit of High-Speed Electric Spindle
}

\author{
Chao Ding, Xiangyang Qi, Zhen Gao, Lei Chang \\ School of Mechanical Engineering, Tiangong University, Tianjin, China \\ Email: dc1995one@163.com
}

How to cite this paper: Ding, C., Qi, X.Y., Gao, Z. and Chang, L. (2020) The Influence of Centrifugal Force on the Interference Fit of High-Speed Electric Spindle. World Journal of Engineering and Technology, 8, 792799.

https://doi.org/10.4236/wjet.2020.84058

Received: October 29, 2020

Accepted: November 23, 2020

Published: November 26, 2020

Copyright $\odot 2020$ by author(s) and Scientific Research Publishing Inc. This work is licensed under the Creative Commons Attribution International License (CC BY 4.0).

http://creativecommons.org/licenses/by/4.0/

\begin{abstract}
The letter presents an analysis of interference fit of the electric spindle at different rotor speeds due to the influence of centrifugal force and provides a theoretical formula showing the relationship of the speed, stress on the rotation speed. The letter also established a finite element model of the interference fit. The study found that as the rotation speed increases, the interference and contact stress between the spindle and the rotor gradually decrease, and the reduced speed is proportional to the square of the rotation speed.
\end{abstract}

\section{Keywords}

Electric Spindle, Interference Fit, Centrifugal Force

\section{Introduction}

As the core component of CNC machine tools, electric spindle has the advantages of compact structure, stable operation and high speed, and is widely used in the field of CNC machining [1]. In order to ensure the machining accuracy and transmission capacity of the electric spindle, an interference connection is adopted between the spindle and the rotor, and the amount of interference will affect the overall cutting performance and processing efficiency of the machine tool [2]. If the interference is selected too large, it will increase the difficulty of assembly between the spindle and the rotor, and even cause the collapse of the mating surface, which will affect the machining accuracy of the machine tool [3] [4]. If the interference is selected too small, the extrusion force between the spindle and the rotor will be small, and sufficient torque will not be transmitted, or the mating surface will slide relatively, which will aggravate the wear of the spindle and affect the service life of the machine tool [5] [6]. When the electric spindle is running at high speed, due to the centrifugal effect, the spindle and the rotor will expand to different degrees, resulting in a great change in the interfe- 
rence fit characteristics between the spindle and the rotor [7] [8]. The letter provides a theoretical formula showing the relationship of the speed, stress on the rotation speed, and also establishes a finite element model of the interference fit [9] [10]. Knowing the interference between the spindle and rotor during the high speed, one can analyze the optimum transmission capacity to improve the processing quality of the parts and hence life of the machine tools.

\section{Principle of Calculation Interference of Electric Spindle}

When the spindle and the rotor are in an interference fit, a positive pressure is generated at the spindle surface, the spindle is compressed, and the rotor is expanded. Since the axial size of the spindle and the rotor is relatively large, and the size along the axial direction is almost unchanged, the axial elongation can be ignored. The matching size of the spindle and the rotor is shown in Figure 1.

The outer surface of the spindle is squeezed, and the inner surface has no load. The boundary conditions are:

$$
\left\{\begin{array}{l}
\sigma_{\rho i}\left(\rho_{1}=b\right)=-P \\
\sigma_{\rho i}\left(\rho_{1}=a\right)=0
\end{array}\left(a \leq \rho_{1} \leq b\right)\right.
$$

where $P$ is positive pressure, $\mathrm{N} / \mathrm{m}^{2} ; \sigma_{\rho i}$ is the radial normal stress of the spindle, $\mathrm{N} / \mathrm{m}^{2}$.

The inner surface of the rotor is subject to expansion, and the outer surface has no load. The boundary conditions are:

$$
\left\{\begin{array}{l}
\sigma_{\rho e}\left(\rho_{2}=b\right)=-P \\
\sigma_{\rho \mathrm{e}}\left(\rho_{2}=c\right)=0
\end{array}\left(b \leq \rho_{2} \leq c\right)\right.
$$

where $\sigma_{\rho e}$ is the radial normal stress of the rotor, $\mathrm{N} / \mathrm{m}^{2}$.

In polar coordinate form, take out the differential body at the angle $\varphi$ and radius $\rho$, and the thickness of the differential body is equal to 1 . Since the spindle and the rotor are both axisymmetric cylindrical sleeves, the tangential displacement and shear stress at the mating surface are zero in the polar coordinate system. The stress distribution of this differential body is shown in Figure 2. The forces on the differential body are projected onto the radial axis of the differential body to obtain a balanced differential equation.

$$
\frac{\mathrm{d} \sigma_{\rho}}{\mathrm{d} \rho}+\frac{\sigma_{\rho}-\sigma_{\varphi}}{\rho}=0
$$

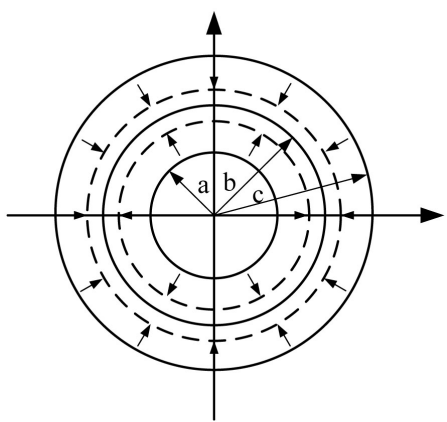

Figure 1. Spindle and rotor matching model. 


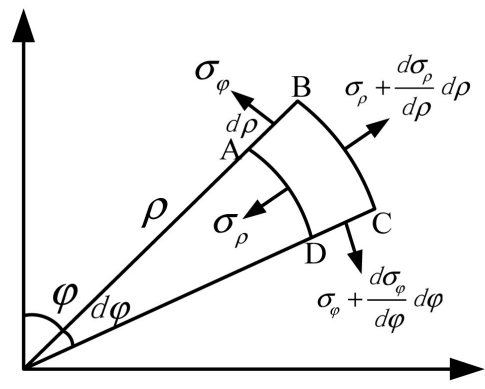

Figure 2. Spindle stress distribution.

According to the literature [11], the relationship between stress and displacement under plane strain is as follows:

$$
\left\{\begin{array}{l}
\sigma_{\rho}=\frac{E(1-v)}{(1+v)(1-2 v)}\left(\frac{\mathrm{d} u_{\rho}}{\mathrm{d} \rho}+\frac{v}{1-v} \frac{u_{\rho}}{\rho}\right) \\
\sigma_{\varphi}=\frac{E(1-v)}{(1+v)(1-2 v)}\left(\frac{u_{\rho}}{\rho}+\frac{v}{1-v} \frac{\mathrm{d} u_{\rho}}{\mathrm{d} \rho}\right)
\end{array}\right.
$$

Substituting (4) into (3), we get:

$$
\frac{\mathrm{d}^{2} u_{\rho}}{\mathrm{d} \rho^{2}}+\frac{1}{\rho} \frac{\mathrm{d} u_{\rho}}{\mathrm{d} \rho}-\frac{u_{\rho}}{\rho^{2}}=0
$$

The general solution of this second-order differential equation is:

$$
u_{\rho}=C_{1} \rho+\frac{C_{2}}{\rho}
$$

According to Formulas (1) (4) (6), the relationship between the stress and displacement of the spindle can be obtained:

$$
\left\{\begin{array}{l}
\sigma_{\rho i}=\frac{b^{2} P}{\left(a^{2}-b^{2}\right)}\left(1-\frac{a^{2}}{\rho_{1}^{2}}\right) \\
\sigma_{\varphi i}=\frac{b^{2} P}{\left(a^{2}-b^{2}\right)}\left(1+\frac{a^{2}}{\rho_{1}^{2}}\right) \\
u_{\rho i}=\frac{\left(1+v_{i}\right) b^{2} P \rho_{1}}{E_{i}\left(a^{2}-b^{2}\right)}\left(1-2 v_{i}+\frac{a^{2}}{\rho_{1}^{2}}\right)
\end{array}\right.
$$

where $\sigma_{\varphi i}$ is the tangential normal stress of the spindle, $\mathrm{N} / \mathrm{m}^{2} ; u_{\rho i}$ is the radial displacement of the spindle, $\mathrm{m} ; E_{i}$ is the elastic modulus of the spindle material, $\mathrm{N} / \mathrm{m}^{2} ; v_{i}$ is the poisson's ratio of the spindle material.

Similarly, the rotor stress and displacement can be determined by the following formula:

$$
\left\{\begin{array}{l}
\sigma_{\rho e}=\frac{b^{2} P}{\left(c^{2}-b^{2}\right)}\left(1-\frac{c^{2}}{\rho_{2}^{2}}\right) \\
\sigma_{\varphi e}=\frac{b^{2} P}{\left(c^{2}-b^{2}\right)}\left(1+\frac{c^{2}}{\rho_{2}^{2}}\right) \\
u_{\rho e}=\frac{\left(1+v_{e}\right) b^{2} P \rho_{2}}{E_{e}\left(c^{2}-b^{2}\right)}\left(1-2 v_{e}+\frac{c^{2}}{\rho_{2}^{2}}\right)
\end{array}\right.
$$


where $\sigma_{\varphi e}$ is the tangential normal stress of the rotor, $\mathrm{N} / \mathrm{m}^{2} ; u_{\rho e}$ is the radial displacement of the rotor, $\mathrm{m} ; E_{e}$ is the elastic modulus of the rotor material, $\mathrm{N} / \mathrm{m}^{2} ; v_{e}$ is the poisson's ratio of the rotor material.

The materials used for the spindle and the rotor are steel materials, and their elastic modulus and poisson's ratio can be considered the same. Therefore, the amount of interference at the mating surface can be determined by the following formula:

$$
\delta_{s}=\frac{2\left(1-v^{2}\right) P b}{E}\left(\frac{c^{2}+b^{2}}{c^{2}-b^{2}}+\frac{b^{2}+a^{2}}{b^{2}-a^{2}}\right)
$$

\section{The Influence of Centrifugal Force on Interference Fit}

When the spindle is running at high speed it is subjected to centrifugal force. The balanced differential equation at this time is:

$$
\frac{\mathrm{d} \sigma_{\rho}}{\mathrm{d} \rho}+\frac{\sigma_{\rho}-\sigma_{\varphi}}{\rho}+\rho_{0} \omega^{2} \rho=0
$$

where $\rho_{0}$ is the material density, $\mathrm{kg} / \mathrm{m}^{3} ; \omega$ is the angular velocity, $\mathrm{rad} / \mathrm{s}$.

According to Formulas (1) (4) (10), the spindle stress and displacement equations are obtained:

$$
\left\{\begin{aligned}
\sigma_{\rho i}= & \frac{(3-2 v) b^{2} \rho_{0} \omega^{2}}{8(1-v)}\left(1+\frac{a^{2}}{b^{2}}-\frac{a^{2}}{\rho_{1}^{2}}-\frac{\rho_{1}^{2}}{b^{2}}\right)+\frac{b^{2} P}{\left(a^{2}-b^{2}\right)}\left(1-\frac{a^{2}}{\rho_{1}^{2}}\right) \\
\sigma_{\varphi i}= & \frac{(3-2 v) b^{2} \rho_{0} \omega^{2}}{8(1-v)}\left[1+\frac{a^{2}}{b^{2}}+\frac{a^{2}}{\rho_{1}^{2}}-\frac{(1+2 v) \rho_{1}^{2}}{(3-2 v) b^{2}}\right]+\frac{b^{2} P}{\left(a^{2}-b^{2}\right)}\left(1+\frac{a^{2}}{\rho_{1}^{2}}\right) \\
u_{\rho i}= & \frac{(3-2 v)(1+v)(1-2 v) b^{2} \rho_{0} \omega^{2} \rho_{1}}{8 E(1-v)}\left[1+\frac{a^{2}}{b^{2}}+\frac{a^{2}}{(1-2 v) \rho_{1}^{2}}-\frac{\rho_{1}^{2}}{(3-2 v) b^{2}}\right] \\
& +\frac{b^{2} P(1+v) \rho_{1}}{\left(a^{2}-b^{2}\right) E}\left(1-2 v+\frac{a^{2}}{\rho_{1}^{2}}\right)
\end{aligned}\right.
$$

According to Formulas (2) (4) (10), the rotor stress and displacement equations are obtained:

$$
\left\{\begin{aligned}
\sigma_{\rho e}= & \frac{(3-2 v) b^{2} \rho_{0} \omega^{2}}{8(1-v)}\left(1+\frac{c^{2}}{b^{2}}-\frac{c^{2}}{\rho_{2}^{2}}-\frac{\rho_{2}^{2}}{b^{2}}\right)+\frac{b^{2} P}{\left(c^{2}-b^{2}\right)}\left(1-\frac{c^{2}}{\rho_{2}^{2}}\right) \\
\sigma_{\varphi e}= & \frac{(3-2 v) b^{2} \rho_{0} \omega^{2}}{8(1-v)}\left[1+\frac{c^{2}}{b^{2}}+\frac{c^{2}}{\rho_{2}^{2}}-\frac{(1+2 v) \rho_{2}^{2}}{(3-2 v) b^{2}}\right]+\frac{b^{2} P}{\left(c^{2}-b^{2}\right)}\left(1+\frac{c^{2}}{\rho_{2}^{2}}\right) \\
u_{\rho e}= & \frac{(3-2 v)(1+v)(1-2 v) b^{2} \rho_{0} \omega^{2} \rho_{2}}{8 E(1-v)}\left[1+\frac{c^{2}}{b^{2}}+\frac{c^{2}}{(1-2 v) \rho_{2}^{2}}-\frac{\rho_{2}^{2}}{(3-2 v) b^{2}}\right] \\
& +\frac{b^{2} P(1+v) \rho_{2}}{\left(c^{2}-b^{2}\right) E}\left(1-2 v+\frac{c^{2}}{\rho_{2}^{2}}\right)
\end{aligned}\right.
$$

When running at high speed, the interference of the electric spindle can be determined by the following formula:

$$
\delta=\frac{(3-2 v)(1+v)\left(c^{2}-a^{2}\right) b \rho_{0} \omega^{2}}{2 E}+\frac{2\left(1-v^{2}\right) P b}{E}\left(\frac{c^{2}+b^{2}}{c^{2}-b^{2}}+\frac{b^{2}+a^{2}}{b^{2}-a^{2}}\right)
$$




\section{Finite Element Analysis}

In the finite element analysis software ANSYS Workbench, the static structural module is used to simulate the interference fit model of the spindle and the rotor. The contact between the spindle and the rotor is frictional contact, where the outer surface of the spindle is the contact and the inner surface of the rotor is the target.

\subsection{The Influence of Centrifugal Force on the Amount of Interference Fit}

When the electric spindle is running at high speed, the spindle and the rotor will expand to different degrees under the action of centrifugal force, which will affect the interference fit characteristics between the spindle and the rotor. Figure 3 shows the radial displacement of the spindle and the rotor at different rotation speeds. In Figures 3(a)-(c) figures represent the radial displacement of the model at different rotation speeds.

It can be seen from Figure 3 that when the interference between the spindle and the rotor is kept constant, as the speed increases, the radial displacement of the spindle on the mating surface gradually decreases, and the radial displacement of the rotor on the mating surface increases. The radial displacement inside the spindle gradually decreases as the rotation speed increases, while the

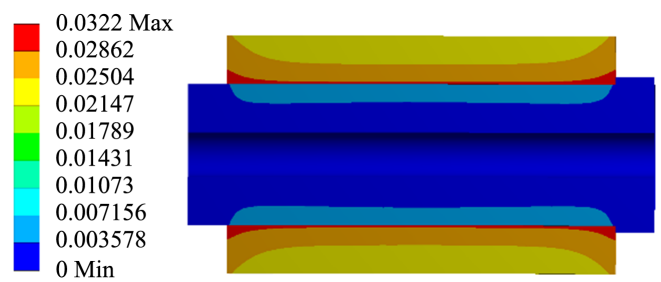

(a)

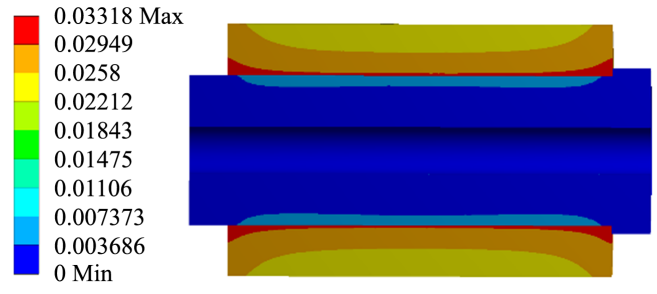

(b)

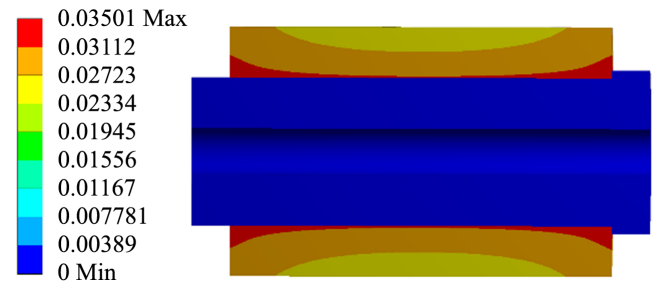

(c)

Figure 3. Radial displacement of spindle and rotor at different rotation speeds. (a) Radial displacement at $5000 \mathrm{r} / \mathrm{min}$; (b) Radial displacement at 10,000 r/min; (c) Radial displacement at $15,000 \mathrm{r} / \mathrm{min}$. 
radial displacement inside the rotor gradually increases as the rotation speed increases. The relationship between the radial displacement of the spindle and the rotor on the mating surface and the rotation speed is shown in Figure 4. In Figure 4, the $\mathrm{X}$-axis represents the rotation speed, and the $\mathrm{Y}$-axis represents the displacement. The blue line represents the rotor displacement; the pink line represents the spindle displacement; the red line represents the reduction of the amount of interference fit between the spindle and the rotor.

When the rotation speed is $1000 \mathrm{r} / \mathrm{min}$, the interference reduction is only $0.043 \mu \mathrm{m}$, which is completely negligible; when the spindle speed is 10,000 $\mathrm{r} / \mathrm{min}$, the interference reduction is $4.3 \mu \mathrm{m}$. The reduction in interference between the spindle and the rotor is proportional to the square of the rotation speed. When the interference is reduced too much, relative sliding or even loosening will occur between the spindle and the rotor, resulting in insufficient torque transmission capacity of the electric spindle, and aggravated spindle wear, which affects the processing quality of the parts. Therefore, in high-speed operation, in order to ensure that the electric spindle has excellent transmission capacity and high speed, it is necessary to increase the interference between the spindle and the rotor to eliminate the influence of centrifugal force.

\subsection{Influence of Centrifugal Force on Contact Stress}

Due to the centrifugal force when the electric spindle is running at high speed, it will offset some of the compressive stress generated by interference, thereby reducing the contact stress between the spindle and the rotor. Figure 5 shows the equivalent stress cloud diagram at different rotation speeds. In Figures 5(a)-(c), figures represent the equivalent stress cloud diagram of the model at different rotation speeds.

Figure 5 shows the behavior of the stress on the rotor. The stress on the inner surface is at large and it gradually reduces from the mating surface to the outer surface of the rotor. Similarly, the stress on the outer surface of the spindle is less than the stress on the inner surface of the rotor, and it gradually increases from the outside to the inside. Because the spindle is more compact in structure and

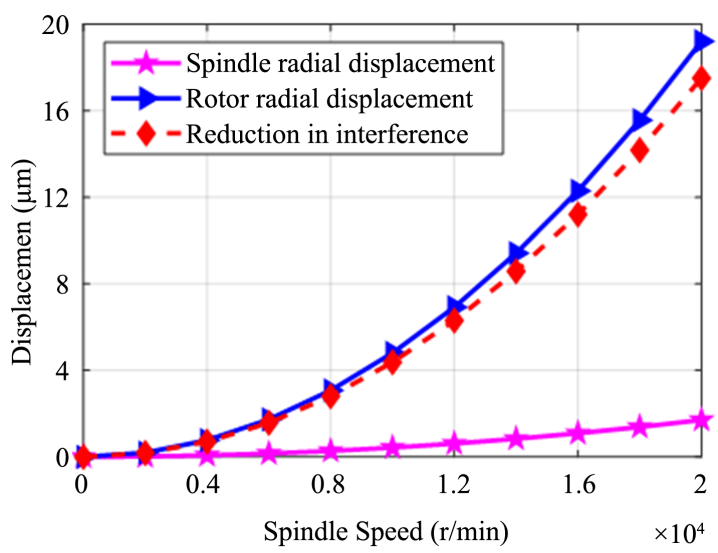

Figure 4. The relationship between speed and displacement. 
has less deformation space, most of the stress is reflected on the rotor. As the rotation speed increases, the stress of the spindle at the mating surface gradually decreases, and the internal stress also gradually decreases. The stress of the rotor at the mating surface increases with the increase of the rotation speed, and the internal stress also increases as shown in Figure 6.

It can be seen from Figure 6 that the contact pressure between the spindle and the rotor decreases as the rotation speed increases. The higher the rotation speed, the faster the contact pressure between the spindle and the rotor decreases.

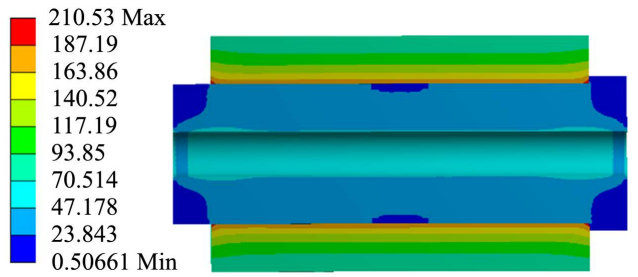

(a)
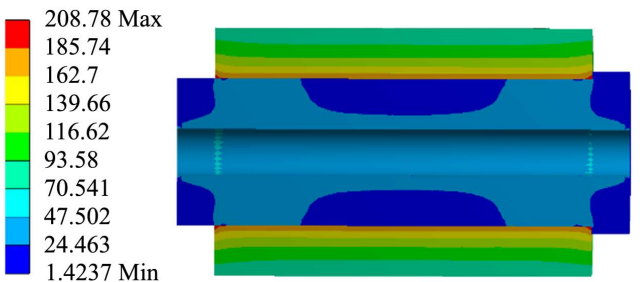

(b)

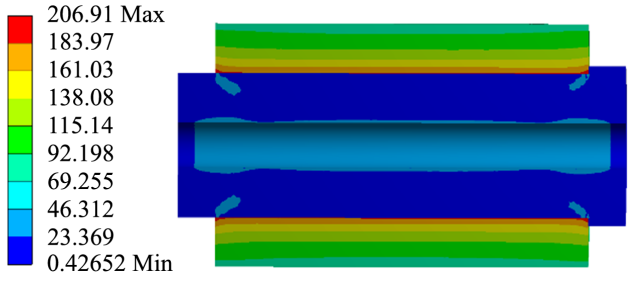

(c)

Figure 5. Equivalent stress cloud diagram at different rotation speeds. (a) Equivalent stress cloud diagram at $5000 \mathrm{r} / \mathrm{min}$; (b) Equivalent stress cloud diagram at 10,000 r/min; (c) Equivalent stress cloud diagram at 15,000 r/min.

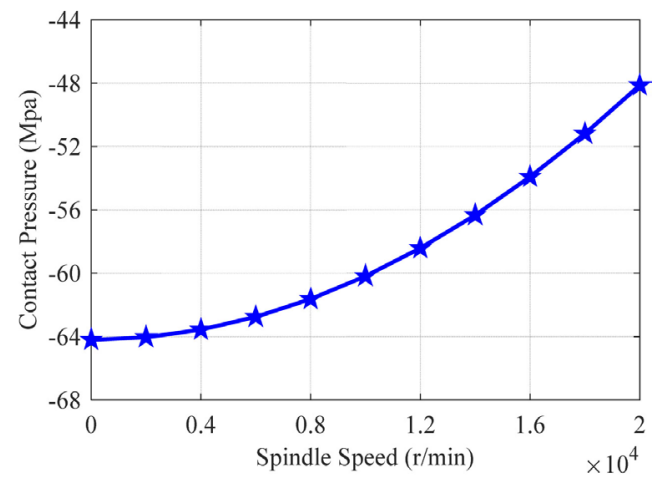

Figure 6. Relationship between rotation speed and contact pressure. 


\section{Conclusion}

When the electric spindle is running at a low speed, the coordination characteristics between the spindle and the rotor are affected by centrifugal force very little and can be ignored. When the electric spindle is running at high speed, the coordination characteristics between the spindle and the rotor are greatly affected by the centrifugal force. As the rotation speed increases, the interference and contact pressure between the spindle and the rotor decrease, and it is proportional to the square of the rotation speed. Therefore, the influence of centrifugal force on the interference fit of the electric spindle must be considered.

\section{Conflicts of Interest}

The authors declare no conflicts of interest regarding the publication of this paper.

\section{References}

[1] Wu, Y.H. (2006) Motorized Spindle Unit Technology of CNC Machine Tool. Machinery Industry Press, Beijing.

[2] Ma, P., Zhang, B.L. and Li, D.N. (1999) Calculation of the Interference Fit for the Electro-Spindle of High Speed Machine Tool. Modular Machine Tool \& Automatic Manufacturing Technique, No. 7, 24-29.

[3] Zhou, C.H., Chen, J. and Tang, C. (2019) Analysis of Interference Fit of Electric Spindle. Machinery, 57, 39-41.

[4] Qiao, W.Y. (2017) The Study of Connecting Rod Bushing Interference Based on Workbench. North University of China, Taiyuan.

[5] Ma, P., Mo, D.Y. and Wu, G.R. (2013) Effects of Step Dynamic Interference Fit on Transmitting Torque Capacity of Motorized Spindle. China Mechanical Engineering, 24, 2933-2938.

[6] Gu, J.J., Cui, Z.X. and Li, X.M. (2008) Design and Verification of the Interference Fit for the Electro-Spindle. Mechanical Engineering \& Automation, No. 4, 161-163.

[7] Zhang, X. and Lu, C.H. (2010) The Effects of Centrifugal Force on Interference Fit between High Speed Spindle and Bearing Inner Ring. Manufacturing Technology \& Machine Tool, No. 9, 39-42+46.

[8] Sun, L.P., Pan, G., Yang, L.P. and Cui, H.F. (2018) Performance Analysis of High-Speed Rotating Shaft Interference Fit. Coal Mine Machinery, 39, 68-71.

[9] Bai, Z., Ma, P. and Lin, Q.W. (2003) The Application of Finite Element Analysis Method to Verify the Stepped Surplus Joint of High Speed Motorized Spindle. Modular Machine Tool \& Automatic Manufacturing Technique, No. 12, 3-4+6.

[10] Zhang, S., Ai, X. and Liu, Z.Q. (2004) FEM-Based Study on Interference Fits in High-Speed Rotatory Spindles. Mechanical Science and Technology for Aerospace Engineering, No. 1, 15-17+24.

[11] Xu, Z.L. (2006) Elasticity. Higher Education Press, Beijing. 